\title{
SOME PRELIMINARY THOUGHTS ON THE HUMAN DEMANDS OF AUTOMATION*
}

\author{
BY
}

\author{
A. T. WELFORD \\ From the Psychological Laboratory, University of Cambridge
}

(RECEIVED FOR PUBLICATION JULY 11, 1957)

It has often been suggested that some of the lines of work developed during and since the war in relation to Service problems can be directly applied to automation: studies for example, of responses to rapid series of signals, of situations requiring vigilance, and of displays and display-control relationships-in short, the stuff of which the psychological aspect of ergonomics has in large measure hitherto consisted. It would seem, however, that these studies are directly relevant in only a very limited way. Industrial conditions from this point of view are more leisurely and predictable than those in the Services, so that rapid response is seldom needed except in rare circumstances, as, for example, in sudden emergencies. Vigilance situations are of types which have in the past shown relatively little problem in that they do not involve the observation of short-duration signals occurring at random, but of watching developing trends in a situation where signals for action do not quickly come and go but gradually increase in magnitude until they are dealt with. Men can get used to almost any display conditions and display-control relationships that are not so obviously wrong that they have already been considered from an ad hoc point of view by management and engineers. There is still a great deal of research to be done on these matters for industry has not managed to solve all its own problems in this area, or indeed to realize them, but solving them would not remove many

*This paper derives from a survey by Miss H. M. Clay, Dr. E. R. F. W. Crossman and the writer, which is being undertaken for the Department of Scientific and Industrial Research and sponsored by the D.S.I.R. and M.R.C. Joint Committee on Individual Efficiency in Industry in an attempt to assess in terms of human skill the demands of work normally included under the heading of automation. The word " skill " is used here in a somewhat broader sense than commonly in industry, to cover not only recognized trade and craft work but any human performance which can show an " expert" quality. The paper represents an attempt at orientation following a study of the literature and a number of factory and other visits, both viewed against a background of general experimental psychology. It was read at a Symposium held by the Ergonomics Research Society at Bristol on April 8-11, 1957. of the main human problems of operating and maintaining automatic machinery.

The Service work is significant for our present purpose, however, to the extent that it implies, when taken in conjunction with more fundamental studies, certain principles of operation of the human organism which may produce different but, nevertheless, important results in industrial work. To put it another way, if we wish to transfer knowledge gained from research done in a Service context to industry, and vice versa, it is necessary to go, not directly but via consideration of the human mechanisms involved.

We shall here consider a number of factors which appear to be important from this point of view and have implications for the design of automation equipment.

\section{Limited Human " Channel Capacity"}

Many researches have now shown well nigh conclusively that the human organism behaves as a single channel of limited information transfer capacity. It can receive input continuously over many channels and give a continuous output over various effectors, but between these there appears to be a single channel dealing with only one transfer from input to output at one time. It is in this channel that limitations on speed of work commonly occur, although in certain circumstances they can arise in other parts of the chain of mechanisms, for example, in the fact that the eyes can only see one small part of a field in foveal vision at a time, or that we have only two hands with which to carry out manual operations.

All forms of automation reduce the number of actual manipulative movements the operator is required to make. The load upon this single channel which results from the need for fine control of movements is therefore also reduced. In so far, however, as there is an increased need for watching and 
listening to what plant or machinery is doing, automation may actually increase the total informational load upon the operator. For instance, on a line of transfer machines the operator who is loading may have a relatively simple manipulative task but will, nevertheless, be very fully employed in observing that the machine is working properly. The load upon him will not so much consist in looking at the actual control panel, which, in examples seen, does not appear difficult to deal with, as in listening to how the machine is functioning-information probably too subtle to be represented on a panel without unduly elaborate sensing devices.

The limitation upon human channel capacity will have its most serious effects where work is paced by some external agency. Contrary to some statements which have been made, all process control operations are paced in the sense that signals for action are liable to arise at times which are not entirely of the operator's own choosing and responses have to be made within time limits. The pacing varies greatly in severity, however, from conditions where processes are remotely but entirely humanly controlled, in which the pacing may be far more exacting than that of a conveyor line, to conditions where the operator's task is almost entirely to monitor a self-regulating set of machinery, taking action only in an emergency. Except in an emergency this latter kind of work is virtually unpaced. Two points need to be made about such conditions. First, however slow the pacing is normally, if signals for action can occur at random and demand attention within very short time limits, there is inevitably a danger that the operator will upon rare occasions be overloaded and that in consequence necessary action will be omitted and an accident result. The events in any actual process are of course far from random; but it seems worth while consciously to build into such a system means of ensuring that the most sudden and unfortunate emergency possible could never present the operator with a situation in which he would not be able to take the necessary action in time.

The second point is that factors in some way related to speed of work have been known for a long time to produce very different subjective effects in the human operator. We can perhaps distinguish four: (1) If the rate is very fast, the operator shows signs of "strain". (2) Rates somewhat slower than this seem to fall within an optimum zone in which strain is absent and the operator's interest is ongaged and well maintained. (3) A rate still slower appears to lead to feelings of boredom and to an unwillingness to do the task. (4) An even slower rate seems to restore tolerable conditions because most of the operator's time is spent doing something other than his main task, for example, reading a book or talking to his neighbours. The precise nature of the speed factor involved has not been determined. It cannot be the number of responses required, nor can it be amount of sensory stimulation. A more likely factor on the basis of present knowledge is " information " in the information theory sense of the term. Objectively, too fast a rate gives rise to tenseness in the subject, hurrying, inaccuracy, and possibly omission of some of the required actions. The changes of behaviour and efficiency which accompany boredom are less well known and more research is needed.

It is a reasonable inference from studies of repetition workers that individual differences exist in just what rate produces what effects: in particular, the optimum becomes lower with diminishing intelligence. The best way of dealing with a boring job of process control might therefore appear to be to employ unintelligent people. This would, however, often mean that the operators were not intelligent enough to take such action as might be necessary from time to time, especially in an emergency. If, therefore, optimum conditions are to be maintained, one of three courses appears to be required: either (a) some strictly unnecessary work has to be added to the task-in some plants operators have periodically to record readings on the dials they are watching, although the data are of no use except as a check that the dials are being inspected occasionally; or (b) such operations need to be deliberately designed for evenness in the amount of intelligence they require at different periods of a working spell; or (c) two people must be employed-one to watch and one to be called in if intelligent action is thought likely to be required.

Before leaving considerations of speed, two further points need briefly to be mentioned. First, work relating time required to make choices to the number of potential alternatives has made it clear that the larger the number of possible signals that have to be watched for, the longer it is likely to take to identify any one when it arrives. Thus a simple increase in the size and complexity of the machine or plant that an operator has to supervise may make its minding more difficult. Secondly, the time taken to deal with signals is increased if they have to be discriminated against a confusing background of other signals or " noise" in the information theory sense: thus the presentation of too much data may be as deleterious to good performarce as the presentation of too little. We may here note that one of the uses of computers which has been recommended is for the production of information for management in a digested form, enabling them to see easily facts which if not in such reduced form would involve 
the inspection of an impossibly large array of figures.

\section{Rules of Action}

The principle that lies behind the results of studies of display-control relationships is that for any job it is necessary to learn and retain a set of " translations" between what we see and what we do. With direct hand actions or hand tools these translations are reasonably straightforward and are learned in the course of manipulating toys at an early age. All forms of mechanization and automation tend to replace these simple relationships with others which are not learned so early and have some element of "arbitrariness" about them. Work on relationships of this kind has shown that some are more in line with expectations than others, presumably because they bear some recognizable similarity to those met with previously, but that almost any set can be learnt and used moderately well provided it is consistent within one machine and from one machine to another used by the same operator. Confusion is likely to arise, however, if inconsistent relationships are required in the same job or between different jobs done by the same man. The confusion may possibly tend to disappear with long experience, but inconsistencies seem likely to lengthen training periods and to remain as a source of confusion on rare occasions for a long time after they have ceased to be troublesome from day to day.

The requirement of consistency and the need it implies for rigid standardization is worth stressing, especially at the present time, because there is an unusual opportunity for manufacturers to adopt and users to demand that certain conventions be rigidly adhered to in the new equipment likely to be installed in the next few years. To achieve standardization will not be without its problems because there are still a number of questions in need of solution before complete standards can be definitely laid down.

The same principle supports the view that standard unit construction, especially of electronic apparatus, would make for easier maintenance and enable lower grade or less trained operatives to do the work. Such construction might for this reason be worth while, even at the cost of using a considerably larger number of components. The principle indicates perhaps with even more force the desirability of anticipatory maintenance according to rule instead of maintenance by repair following breakdown.

\section{Conceptualization of the Process}

Probably the most important aspect of the principle we have been discussing is, however, that display-control relationships seem to provide what can be regarded as, in a very literal sense of the word, elementary examples of conceptualization of the process being controlled. There is little doubt that one of the most important abilities of the operator on a complex machine is, and will to an increasing extent become, that of having some " picture" or " model " in his mind of the machine and of its work. The picture or model need not be as complete as that which an engineer would have and need not even be accurate, provided it enables the operator to do two things: first, to give meaning to the sight and sounds of the machinery he is controlling, and secondly to monitor by observation of results what is going on in parts of the machine or of the plant he cannot actually see.

Just what is involved in such conceptualization has never been fully formulated, but it appears to involve two processes, about each of which something is already known and further close study would be worth while.

It seems to require the coordination into a " scheme" or "frame of reference" of pieces of data culled at different times in the course of experience. We all use schemes of this general kind in order, for example, to maintain spatial orientation and find our way about. It is well known that individuals differ in their ability to build and maintain such schemes and that circumstances may hinder or facilitate their formation. Some practical steps towards making conceptualization easier have already been taken in the diagrammatic layout of instrument panels, but clearly more needs to be known, especially with regard to training men to monitor machines which do not readily lend themselves to such display methods. In any case, it must be emphasized that good instrument layout can deal with only a part of the whole problem, which may perhaps be epitomized by asking the question, " How do we acquire the ability to ' sense' whether our car is running well or not and how can we train someone else to do the same?"

The second aspect of conceptualization which is important in this context is the ability to manipulate data in the abstract-to imagine events which are not seen or which, as in the case of electronic apparatus, are unobservable. Such ability enters largely into so-called intelligence tests, and it is perhaps understandable that Vernon (1947) in his studies of the intelligence test scores of British naval personnel during the war found that electrical artificers attained scores higher on average than those of any other rating. They were a little higher than those of R.N. executive officers and Fleet Air Arm observers and pilots and were exceeded only by those of R.N. engineering officers. There are not many people with mental ability at this level and 
it is important therefore to consider ways in which the working of a machine can be represented to the operator in as concrete a manner as possible. At the same time, however, we should recognize that long experience with the same machine-implying stability of employment-is likely to enable a man to achieve an understanding of this one particular machine which he would be unable to attain on a more general basis.

A few other points may be more briefly mentioned.

\section{Anticipatory Action and Prediction}

When a man forms part of a control loop, the time relations are important between the taking of action and its becoming effective. If the time is long the man must either predict the effects of his actions so that he makes a "ballistic" adjustment to his controls which will produce the correct results after the system has settled down, or he will tend to oscillate in a series of more or less violent overcorrections. If using velocity controls he may also be faced with the need to remember action taken at one moment with a view to taking cancelling action at a later time, often, if the process is slow, after other actions have been taken during the interval. The load upon his short-term retention in such circumstances can be severe, with consequent danger of adjustments being forgotten.

Where possible, it would be worth while to build into the apparatus means of avoiding the need for the operator to predict and remember in this way, for example, by giving indication immediately of what the eventual effect of his action will be when the system has settled down, or of storing data for him by devices such as cursors on scales which can be set to indicate required readings so that discrepancies can easily be noted. To do this might often require a degree of definition of the task greater than had yet been undertaken, especially when many variables have to be controlled simultaneously. Engineers and management seem often to have despaired of the task of formulating processes sufficiently to enable predictors or automatic control to be used. Instead, a carefully selected operator is employed who manipulates controls to produce a more or less satisfactory end-product by some means akin to an art, which he obviously does not fully understand and certainly cannot adequately formulate in words. The definition of such skills-and they are skills in a very real sense of the word-is not at present possible, although a few tentative suggestions can be made. Perhaps the most significant of these is that as the number of variables is increased, the difficulty of the task seems, for a variety of reasons, to increase disproportionately. The control of only one or two variables in a complex task might therefore be expected to reduce the difficulty for the operator much more than would be predicted from a study of a task involving these one or two variables in a simpler context.

\section{Changes in Nature of Skill Resulting from Automation}

Skilled action in the broad sense of the term is of many different kinds; but probably the most difficult components of manipulative tasks, as practised by semi-skilled operatives on work such as light assembly and which enter into skilled craft operations, are the making of precisely graded ballistic movements. It is characteristic of automatic processes that fine motor control is no longer required and in this respect they seem to make the operator's task much easier. Fine motor actions appear to need continual training if a high level of performance is to be maintained: the "rustiness" of a tennis player at the beginning of the season or of a concert musician out of practice, has its industrial counterparts.

There remains a requirement to perform actions in the correct order, but with many automatic machines, especially, for example, with tape-fed machines, the order of actions is worked out once for all at leisure and does not have to be carried in the mind of the operator the whole time. For him, the simpler routine of loading the machine is sufficient, and this can be made common to what would before have been tasks involving many different routines.

Automation may also tend to replace fine subjective judgments, such as of the colour of molten metal, which may take years to acquire and, like fine graded movements, require continual practice to maintain.

Viewing these changes together it appears that automation tends to conserve skill in the sense that it reduces the need for traditional components of skill to be used repetitively, and leaves men to use their unique human capacity for dealing with the complex and the variable in primary construction, in emergencies, and to some extent in maintenance. Even in this last we may doubtless look forward to a kind of "meta-automation" where such items as tool grinding will be carried out automatically.

\section{The Operator's Need for Adequate Information}

The fact that the operator of automatic plant is often removed from direct contact with the process he is controlling emphasizes the need to consider thoroughly what information he needs to be supplied with in order to do his work not only accurately but with confidence. Broadly speaking he must have unambiguous indications of what is 
happening and of the effects of his actions, and must also have data which will enable him to be sure that his apparatus is at all times working properlyabsence of significant events in the process should not be signalled merely by absence of any indication.

The indications should be such that they are clear and do not require any elaborate process of inference or piecing together of data from several sources. Ideally every fundamentally distinct event should be separately signalled. What constitutes a " fundamental distinction" in this sense is often intuitively obvious although it is not precisely definable. To do so in general terms a great deal of research would be needed, the contributions of which would have considerable theoretical as well as practical significance.

\section{Re-training of Middle-aged Operatives}

One of the most important, although probably temporary, problems introduced by automation is due to the fact that men and women re-deployed as a result of changes of process and work method will probably be middle-aged and in consequence have some difficulties in acquiring new knowledge. We cannot enter into this matter fully here, but the evidence is that the essential difficulty experienced by middle-aged people when learning new tasks lies not so much in learning and retention as in the actual process of taking training, so that suitable methods of training for people in middle age seems likely very substantially to reduce their problems. The devising of new methods of training for this purpose seems to be an urgent need.

\section{Responsibility}

Of the human qualities having rarity value which are commonly suggested as important for operatives in an automatic plant, the most frequently cited is a " sense of responsibility". This is, however, a vague term which needs further definition before it can guide the selection of men or the design of jobs. In the present context it seems commonly to cover three requirements. (a) The first is reliability in the sense that behaviour should be predictable and not show " random " variations due either to insufficient attention to the job or to taking action other than what is prescribed, whether by error or in an attempt to improve upon established routine. Ensuring reliable operators is probably, in the present state of knowledge, a matter of selection, probably by means of trial on the job. (b) The second requirement is that whatever has to be done shall be done with sufficient accuracy as quickly as possible. This requirement might in fact be made very much less pressing by systematic and thorough application of engineering psychology principles to the simplifica- tion of the job. (c) A requirement of a somewhat different kind is a willingness to recognize one's place in a hierarchy without the corresponding willingness to " pass the buck". Both this and the preceding quality raise problems of motivation, incentive, and will to work. Doubtless recognized factors such as knowledge of results, understanding of the part played by the operation in the final product, payment methods, and so on, will have some application still under automatic conditions. We may suspect, however, that it will be necessary sooner or later to regard these aspects of responsibility as attitudinal matters, or, to use an older word, " moral " matters and to consider their implications for the status of the operator. They appear to be characteristic of what one might call a vocational or professional approach to work and to raise the possibility that operators should be given salaried positions.

\section{Satisfaction with Work}

Discussions of the effects of automation frequently raise the intangible, subjective and therefore somewhat suspect, concept of work satisfaction. It must be recognized that degree of work satisfaction is one of several end-products of the factors already discussed and should not be considered apart from them. Other factors also contribute, however, and must be taken into account when making an overall survey of the human demands of automation. The suggestion is often made that the monotonous conditions and relative isolation of workpeople from one another in an automatic factory may cause dissatisfaction. On the other hand, attention to the points raised earlier seems likely at least to minimize dissatisfaction if not indeed to make work more satisfying than it had been before. Two other subjective factors seem also to be worth mention and research study. First, the control of powerful and highly productive machinery seems likely to have an inherently satisfying effect, possibly because of the high ratio of result to effort obtained by its use. As against this, large and powerful plant may be dangerous in the sense that failure to operate it correctly may lead to serious results and factors not under the operator's control may produce a danger of accidents. The risk may be very small but there are suggestions that when hazards of this kind are recognized to exist the effect over many years may lead to an unwillingness to continue with the job, or alternatively to signs of strain. Secondly, many people seem to derive their chief satisfaction from the creation of something unique, regardless of the effort involved. Automatic plant appears at first sight to diminish this second type of satisfaction. It is likely, however, that many of the new jobs will have something of this quality. For example, those 
designing programmes for computers seem to derive satisfaction from the fact that a new programme, once made, is in a sense a unique and enduring product, capable of continued use without having to be created again. We may perhaps add that the automatic production of necessities and " essential luxuries" may well bring greater opportunities for the artist and craftsman in certain areas of production not covered by automation, and bring their work into increasing demand.

The indications of the various points outlined in this paper are by no means mutually compatible, so that the machine and process designer will often have to balance one inconsistent requirement against another. The result-that each case needs careful individual consideration-does not mean, however, that general principles cannot be formulated. It does mean some rather exacting analytical thinking on the part of those who seek to understand the psychological problems of automation.

\section{Summary}

An attempt is made to outline some of the factors in human skilled performance which seem, from the standpoint of experimental psychology, likely to be of importance in work with automation equipment.

Automation appears to reduce the need among operators for actual manipulative skill but to place increased emphasis upon a number of more intellectual capacities. These demands are discussed in terms of (1) the limited " information channel capacity" of the human organism with the suggestion that both over- and underloading of this capacity are to be avoided; (2) the need for consistency in the relationships between display and control; and (3) the coordination of data from many sources and arriving at different times, and the conceptualization of the task which appears to be necessary for adequate performance.

Briefer discussion is included of the problem of what constitutes adequate information in displays, of the most suitable routines for repair work, of the urgent need to devise methods of re-training middle-aged operatives who have to be redeployed, of what is meant by " responsibility" on the part of operatives, and of the likelihood that automation will result in gains as well as losses in work satisfaction.

\footnotetext{
Vernon, P. E. (1947). Amer. Psychol., 2, 35.
} 